\title{
Smoking as an Environmental Health Problem
}

\author{
Prof. Dr. M. Ihsan Karaman, MD
}

International Journal of Human and Health Sciences Vol. 03 No. 03 July'19 Page : 123-126

DOI: http://dx.doi.org/10.31344/ijhhs.v3i3.89

The use of tobacco and tobacco products, particularly cigarettes, causes many diseases that threaten human health. Despite all the work carried out on a global scale, the use of tobacco and tobacco products remains an important public health issue.

In addition to the direct effects of cigarettes on human health, such as cancer, immune system and autoimmune disorders, premature deaths, type 2 diabetes, lung disorders, heart attack and stroke, passive smoking-related complications and pregnancy complications, they cause direct harm on the environment and the ecosystem as well, such as deforestation, generation of huge amounts of toxic waste, air pollution through industrial production process and farming, soil and land pollution through farming and from cigarette butts, air pollution through smoking, contamination of waterways due to cigarette butts, health impacts on aquatic fish, health impacts on pets and forest fires and wildfires.

Therefore, we have to focus not only on the direct effects of cigarette smoking on human health but also its harmful effects on the environment and the ecosystem. In this manner, we can see and show damages broadly and can develop extensive prevention strategies. Instead of using ordinary slogans such as "Smoking is harmful to health and causes damage to lungs" and studies emphasizing only on the individual harms, we have to develop preventive activities against the overall effects of tobacco on the environment and the ecosystem.

Although we have seen and heard many times the health hazards of tobacco and tobacco products from public spots and warnings on the package, we have to pay attention to how much the entire ecosystem is affected from them.

As a medical doctor and an international activist against addiction including tobacco, in the NGO sector, I can write a long separate article on the effects of smoking on health. However, in this editorial,I am going to concentrate on its harmful effects on our environment.

\section{Effects of Cigarette Smoking on Environment}

These direct effects of individual cigarette consumption which threaten the human health; are further increasing due to the harmful outcomes of smoking on the environment and ecosystem. Namely; tobacco smoke contain more than 4000 chemicals, of them many are toxic and carcinogen, and their use causes environmental pollution by releasing toxic wastes to the atmosphere. Likewise, cigarette butts pollute the environment; poisonous chemicals in the remnants of butts are infiltrated into the soil and waterways, causing soil and water pollution, respectively. Animals that contact or ingest toxic substances in cigarette residue / cigarette butts and sucking plants are also adversely affected by cigarette smoking.

More than 5 trillion cigarettes are smoked worldwide each year, and all of them are disposed of in some manner. Smoking globally emits nearly 2.6 billion kilograms of carbon dioxide and 5.2 billion kilograms of methane into the atmosphere each year due to the methods used to manufacture cigarettes and other tobacco products.

The high scores of pesticides, fertilizers and other chemicals used in the cultivation of tobacco introduce volumes of hazardous pollutants to the water and soils. These chemicals accumulate and eventually hamper the fertility of the soils and make the lands unsuitable for supporting any other crop. Additionally, a publication even indicated that an hour cigarette-manufacturing unit needs about 4 miles of paper for rolling and packing which translates to the destruction of one tree for every 300 cigarettes made. Thousands of trees are cut down for cigarette paper and its package. Most of the ingredients present in cigarette butts, on the other hand, are non-biodegradable and take years to break down. On top of that, butts are also often eaten by birds, fish, and other animals, who can choke on them or be hurt from the poisons they contain.

Correspondence to: M. Ihsan Karaman, MD, PhD, Professor of Urology, President FIMA, President International Federation of GreenCrescents, Istanbul, Turkey. Email: mikaraman@hotmail.com 
As such, it's not only the cigarette smoke that causes manifold impacts on people and the environment but also the cigarette butt and other wastes released during the entire production process of cigarettes. Interestingly, when people hear about cigarette smoking, they often think of the health risks it has on the human body. Many fail look at the critical side topic which pertains to how it harms the environment. However; the impact of cigarette use on human health and environment should be considered together.

The environmental lifecycle of tobacco can be roughly divided into four stages:

(i) tobacco cultivation and curing;

(ii) product manufacturing and distribution;

(iii) product consumption;

and (iv) post-consumption waste.

By taking these stages into consideration, environmental and health issues should be identified together and policy recommendations / prevention strategies should be established.

Here, we describe the environmental and health concerns at each of these stages and propose recommendations for policy-makers.

\section{Tobacco Cultivation and Curing}

The production of tobacco and tobacco products causes widespread environmental degradation around the world. It begins with the preparation of land for tobacco cultivation and carries through the life of these products as they are manufactured, marketed and consumed. While the cultivation of tobacco causes deforestation, the manufacturing process of tobacco products releases many toxic chemicals. The waste from production - much of it toxic - and disposal of packaging and cigarette butts pollute our fragile ecosystems.

The entire process of cultivating, curing, and transporting tobacco needs the use of large amount of chemical and other toxic materials. At the same time, the production process generates huge amounts of wastes such as harmful chemical pesticides and fertilizers. One of the habitually used substances in the production process is known as Aldicarb. It's highly toxic to humans, plants and animals and can seep into waterways and intoxicate the soil for several years. Other toxic wastes generated from cigarette production include Dithane DF, Imidacloprid, 1, 3- Dichloropropene, Chlorpyrifos and methyl bromide which can harm plants, humans and animals.

With the rapid increase in global tobacco consumption in the late 20th and early 21 st centuries, the demand for tobacco leaf increased dramatically. This has led to a correspondingly dramatic increase in the amount of land dedicated to tobacco production. Tobacco industry, which grew rapidly with the increasing demand for tobacco products, has begun to harm the environment more than ever with the chemical wastes it produces. Tobacco consumption, on the hand, continues to harm both human health and environmental health and the ecosystem increasingly in various ways. As a consequence of expanded tobacco agriculture, there are short-term economic benefits for some farmers, but there will be long-term social, economic, health and environmental detriments for many others.

Frequently, farmers clear forested land that is agriculturally marginal to grow tobacco- often by burning - and/or harvest wood for curing. Typically, after only a few seasons, the land is quickly abandoned and becomes unusable, leading in many cases to desertification. Not only does burning generate vast amounts of land, water and air pollutants, much of this land is cleared from carbon dioxide-absorbing forest cover. As a result, tobacco cultivation is exacerbating greenhouse gas levels.

Tobacco cultivation is typically one of the most chemically-intensive crops. Because much of the land on which tobacco is grown is marginal, most farmers heavily use inorganic chemical fertilizers to promote growth and herbicides to mitigate competing weeds. Because tobacco is typically grown as a monocrop, it is also particularly vulnerable to pests and most farmers heavily use pesticides (to protect tobacco). Furthermore, in many countries, there is evidence that farmers continue to use chemicals that are restricted or banned in most higher-HDI (human development index) regions, such as the European Union.

\section{Product Manufacturing and Distribution}

Tobacco products generates many environmental hazards in the manufacturing process as well as in consumption. The tobacco manufacturing process and all activities that use tobacco produce solid or liquid wastes with high concentrations of nicotine. In addition, tobacco manufacturing produces chemical wastes, including nicotine, in the manufacture of cigarettes, cigars, smokeless tobacco, and other products. Many of these waste products are potential environmental hazards.

The tobacco product manufacturing process generates vast amounts of waste. The last rigorous estimate, from 1995, suggested that the industry produces more than 2.5 million tons of manufacturing waste much of which contains 
nicotine and other dangerous chemicals. As global tobacco production is greater than in 1995, this negative impact can only be higher still today. Once raw tobacco leaf has been grown by a farmer and sold to a manufacturer, it must be processed into a desirable consumer product. To maximize profits, tobacco manufacturers want to make products as attractive and addictive as possible. While intensifying chemicals in the product increase its "quality" and sale, its damage to the environment and human health increases as well and even more. The packaging and labeling of tobacco products is resource-intensive in terms of the paper, plastic and chemicals that manufacturers use. Millions of tons of packaging waste, much of it plastic, ends up as litter or helps to overwhelm landfills around the world. Similarly, the disposal of cigarette waste after consumption causes harm to the environment.

\section{Product Consumption and Post-Consumption Waste}

\section{a. Cigarette Smoke}

Smoking tobacco causes exposure to a lethal mixture of more than 7000 toxic chemicals, including at least 70 known carcinogens that can damage nearly every organ system in the human body. Smoking globally emits nearly 2.6 billion kilograms of carbon dioxide and 5.2 billion kilograms of methane into the atmosphere each year. This provides a clear picture of how smoking alone contributes to climate change. These toxic particles in the atmosphere can hang in the air for hours and it reduces the air quality of the atmosphere. This pollution happening in the atmosphere also causes cardiovascular diseases, impairment in lung function and lung cancer. Globally, 942 million men and 175 million women ages 15 or older are current smokers. Nearly three quarters of male daily smokers live in countries with a medium or high human development index (HDI), whereas half of female daily smokers live in very high-HDI countries. (When we consider these data) When we take this into consideration, we can say that one in every six people causes air pollution with tobacco smoke.

Air pollution is unarguably the greatest environmental catastrophe in the world today. It kills more people worldwide each year than does AIDS, malaria, diabetes or tuberculosis. The most harmful pollution consists of small particulate matter, 2.5 microns in size or less, called PM. These particles are small enough to work their way deep into the lungs and into the bloodstream, where they trigger heart attack, stroke, lung cancer and asthma.
Exposure to secondhand smoke is associated with numerous adverse health effects, even among children and unborn babies, and causes substantial mortality and morbidity globally. In 2016 alone, for example, it caused an estimated 884,000 deaths.

Exposure to secondhand smoke is common in many countries, notably in Asia. In Indonesia and Pakistan, for example, more than $80 \%$ of people are exposed to secondhand smoke in restaurants. However, the implementation of laws developed under the smoke-free airspace greatly affects the level of exposure to cigarette smoke. After implementation of comprehensive laws in Turkey in 2009, exposure prevalence in work-places and restaurants dropped substantially- from $37 \%$ and $56 \%$ respectively in 2008 to $16 \%$ and $13 \%$ in 2012. That is to say, it is possible to decrease passive smoking (second-hand smoking) resulting from cigarette/tobacco smoke with preventive policies! We are able to ensure that. Likewise, we can also make possible the prevention of air pollution caused by tobacco use by remarking the issue and reducing the rates of tobacco product use. Tobacco and tobacco product use do not affect only users and their surroundings. It damages the whole ecosystem.

b. Cigarette butts: Soil, water and other living beings

Cigarette butts are non-biodegradable garbage, toxic when ingested by humans and other living things and leave chemicals and heavy metals in the environment.

Cigarette butts are the most commonly discarded piece of waste globally and are the most frequent item of litter picked up on beaches and water edges worldwide. The non-biodegradable cellulose acetate filter attached to most manufactured cigarettes is the main component of cigarette butt waste and trillions of filter-tipped butts are discarded annually.

Since cellulose acetate filters from cigarette butts and plastic materials are non-biodegradable, they remain in the nature for a long time and accordingly damage the ecosystem. And we can clearly see the wastes of cigarette consumption, that is to say the cigarettes, cigarette packets and gelatins while walking in the streets and on the city pavements, using public beaches or sitting on the beach.

The filters are made of cellulose acetate, sourced from plastic, are photodegradable - can be broken down by UV light but still take an extended period to break down. The ingredients in the filter 
therefore remain in the soil for a long period of time, up to 10 years as estimated by researchers. As long as they are present in the soil, the soil remains polluted.

With 6 trillion cigarettes manufactured annually, about 300 billion packages (assuming 20 cigarettes per pack) are made for tobacco products. Each year, 6 trillion cigarette butts are infiltrated into seas and oceans as a garbage causing a serious threat to marine life. It takes up to 10 years for a single cigarette butt to dissolve completely. These cigarette pieces thrown around not only create a bad scene, but also pose a threat to plants, animals and even ground waters.

Fish have particularly been impacted by cigarettes in countless ways. Whenever cigarette filters find way into water systems, they can be ingested by fish because they resemble fish food like the insects. The filters remain within the fish reducing their stomach capacity, thus affecting their eating habits. The threat of cigarette butts against fish is not limited with this. Research in the US also found that the runoff from just a single cigarette butt can kill a fish in a 1 Liter jar of water. If this is translated into the amounts of the cigarette butts that find their way into water systems, it's more than clear the degree at which fish are impacted every year. Humans are likewise not spared if by any chance they ingest the chemicals by consuming affected fish.

Similarly, the disposal of cigarette waste after consumption causes harm to the environment. In beach clean-up efforts around the world, cigarette butts comprise the largest component of the waste. Lastly, the fires caused by cigarette smoking and butts cause great harm to the environment, beyond their costs in terms of lives lost and direct economic loss. Cigarette smoking is a major cause of both house and forest fires throughout the world. In both the USA and the United Kingdom, cigarettes are the single greatest cause of fire-related deaths. The forests fires started by burning cigarette butts worldwide are countless. About 17,000 people and countless living being worldwide die each year because of fires started by cigarette lighters or discarded burning cigarettes. In terms of property damage, the losses are more than 27 billion US dollars every year.

\section{Conclusion and Suggestions}

The production of tobacco and tobacco products causes widespread environmental degradation around the world. It begins with the preparation of land for tobacco cultivation and carries through the life of these products as they are manufactured, marketed and consumed. After examining these harms with its all dimensions, preventive strategies should be developed.

The WHO Framework Convention on Tobacco Control (FCTC) addresses environmental concerns related to tobacco in Article 18, and the need for alternative livelihoods for tobacco growers in Article 17. However, this issue needs to be addressed in a more comprehensive way.

For this, stronger regulations on tobacco manufacturers can be developed to prevent the tobacco industry from harming the environment. Disposable (single-use) filters--including any biodegradable varieties-- can be banned in order to reduce waste in the manufacturing process. Overuse of paper and plastic products can be prevented to mitigate packaging. Based on the level of the damage caused by the tobacco industry, tax payment can be foreseen.

Tobacco farmers can be guided to find environmentally-friendly alternative crops. Farmers should be also informed to supply and value chains of other locally-grown crops. Extension services and agricultural education for non-tobacco crops can be offered to them. Access to credit for cultivating non-tobacco crops should be provided easily for them. The WHO-FCTC commitment should be applied to governments not investing in tobacco farming. Governments should help to educate the farmers who continue to cultivate tobacco on environmentally friendlier practices. Shortly, governments must help to improve supply and value chains for alternatives to tobacco leaf, and invest in farmers' education/ re-training programs.

However, above all, with awareness studies attention can be drawn to the fact that people have been harming all living being before themselves by using tobacco and tobacco products. You are not only harming your health or the health of your loved ones, but also harming the nature, the environment, and all living being.

In sum, we underscore that while we continue to face a very serious challenge, we have the tools to make an enormous positive difference. 